\title{
关于方程 $|\operatorname{Aut}(G)|=p^{2} q^{2}$ 的解 ${ }^{*}$
}

\author{
李世荣

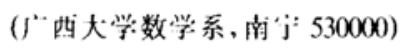

\section{关键词 有限群 自同构群 群阶}

\section{1 结果}

我们关心如下问题: 给定有限群 $G$, 确定有限群 $X$, 使得 $\operatorname{Aut}(X)=G$, 而 $\operatorname{Aut}(X)$ 表示 $X$ 的全 自同构群. Iyer ${ }^{[1]}$ 证明了上述方程的解至多有有限个. 对于任意固定的正整数 $n$, 同样的结论 对方程 $|\operatorname{Aut}(X)|=n$ 成立. $n$ 的某些特殊情形已被研究, Machale ${ }^{[2]}$ 和 Curran ${ }^{[3]}$ 证明了, 对任一 奇素数 $p,|\operatorname{Aut}(X)|=p^{m}(1 \leqslant m \leqslant 5)$ 无解; $\operatorname{Flym}^{[4]}$ 给出 $|\operatorname{Aut}(X)|=2^{5}$ 的全部解; $n=p^{2} q(p$ 和 $q$ 是不 同的素数) 在文献[5] 和 [6] 中被研究, 本文利用文献[7] 的结果, 完整地解决了 $n=p^{2} q^{2}$ 的情 形.

我们用 $r_{1}, r_{2}$ 和 $r_{3}$ 分别表示形如 $4 q^{2}+1,2 q^{2}+1$ 和 $2 q+1$ 的素数, 而 $q$ 为奇素数. 本文的 结果如下:

定理 1 令 $G$ 为有限群, $p$ 和 $q$ 是不同的素数, 那么 $|\operatorname{Aut}(G)|=p^{2} q^{2}$ 当且仅当 $G$ 为下列群之

(a) $C_{n}, n$ 为 $125,250,108$ 或形如 $r_{1}, 2 r_{1} 3 r_{2}, 4 r_{2}, 6 r_{2}, 9 r_{3}, 18 r_{3}$ 之数;

(b) $C_{2} \times C_{2} \times C_{n}, n=7$ 或 9 ;

(c) $S_{3} \times C_{n}, n=7$ 或 9 ,

其中 $C_{n}$ 表示 $n$ 阶循环群, $S_{3}$ 表示三次对称群.

定理 1 的一个直接推论是

推论 1 设有限群 $G$ 满足 $|G / Z(G)|=p^{2} q^{2}, p$ 和 $q$ 同上, 那么 $G$ 有一个外自同构.

群 $G$ 的中心自同构是一个自同构使得它诱导出 $G / Z(G)$ 的恒等自同构. 中心自同构的全 体作成 $\operatorname{Aut}(G)$ 的一个子群, 记为 $\operatorname{Cent}(G) ; G$ 的内自同构群用 $\operatorname{lnn}(G)$ 表示. 设 $H, K$ 为群, 我们 用 $[H] K$ 表示 $H$ 借助 $K$ 的半直积. 凡未加说明的符号都是标准的.

\section{2 引理}

在以下诸引理中, $G$ 总表示一个有限群.

引理 $1^{12]} \operatorname{Cent}(G)=C_{\mathrm{Aur}(G)}(\operatorname{Inn}(G))$.

令 $p_{1}, \cdots, p_{r}$ 是 $|G|$ 的所有素因子, 并令 $G / G^{\prime}$ 和 $Z(G)$ 的 Sylow 分解如下: 
引理 $2^{[8]}$ 若 $G$ 没有非平凡的交换直因子, 那么

$$
\begin{gathered}
G / G^{\prime}=G_{p_{1}} / G^{\prime} \times \cdots \times G_{p_{r}} / G^{\prime}, \\
Z(G)=Z_{p_{1}} \times Z_{p_{2}} \times \cdots \times Z_{p_{r}} .
\end{gathered}
$$

$$
|\operatorname{Cent}(G)|=\prod_{i=1}^{r} \prod_{j=1}^{k_{i}}\left|Z_{p_{i} j}\right|^{r_{i j}},
$$

其中子群 $Z_{p_{i} j}=\left\{z \mid z \in Z_{p_{i}}\right.$ 且 $\left.z^{p_{i}^{i}}=1\right\}, p_{i}^{k_{i}}$ 为 $G_{p_{i}} / G^{\prime}$ 的幕指数 (exponent), $r_{i j}$ 是 $G_{p_{i}} / G^{\prime}$ 的直分解式中 阶为 $p_{j}^{i}$ 的直因子 (循环群) 的个数, 它是一个不变量.

接下来我们给出如下 3 个引理, 它们的证明在这里略去.

引理 3 设偶阶群 $G=P[Q], P$ 为 $G$ 的 Sylow 2-子群, $Q$ 为 $q^{2}$ 阶初等交换群, $q$ 为奇素 数. 还假设 $C_{P}(Q)=1, Z(G)=1$, 那么 $2^{3} \| \operatorname{Aut}(G) \mid$.

引理 4 设 $G$ 是一个极小非幂零群满足 $G / Z(G) \cong A_{4}$ (四次交错群), 那么 $2^{3}|| \operatorname{Aut}(G) \mid$.

引理 5 令 $p$ 为固定奇素数, $G$ 是一个 $p$-群. 如果 $|G / Z(G)|=p^{2}$, 那么 $G=[A] B$, 这里 $A$ 为 交换子群, $B$ 为循环.

\section{3 定理 1 的证明}

假设 $G$ 满足定理的条件, $P, Q$ 分别表示 $G$ 的 Sylow $p$-子群和 Sylow $q$-子群. 由文献[7], $p^{2} q^{2}$ 必定是偶数, 故可设 $2=p<q$. 定理的证明由 3 部分组成, 即

命题 1 若 $G$ 为幂零, 则结论 (a) 和 (b) 成立;

命题 $2 G$ 不可能是无非平凡交换直因子的非幂零群;

命题 3 若 $G$ 非幂零, 且 $G$ 有非平凡交换直因子, 则结论 (c) 成立.

命题 1 的证明不难, 命题 3 的证明由命题 2 及文献[6] 经计算而得, 所以关键是证明命题 2 .

设 $G$ 非幂零且无非平凡的交换直因子, 通过分析 $G$ 的结构, 我们导出一个矛盾.

(1) $|\operatorname{Cent}(G)|_{2} \leqslant 2$ 且 $|\operatorname{Cent}(G)|_{q} \leqslant q$. 这由 $\operatorname{Inn}(G) \cong G / Z(G)$ 非幕零及引理 1 推出.

(2) $Q \triangleright G$.

假设不真, 那么 $G$ 含有极小非幕零子群 $K$ 使得 $K / Z(G) \cong A_{4}$, 而 $q=3$. 从而推出 $P \triangleright G$, 因 此 $G / G^{\prime}$ 具有非平凡 Sylow 3 - 子群. 可断言: $3 \nmid|Z(G)|$. 否则, 由引理 2 及结论 (1) 推出 $G / G^{\prime}$ 的 Sylow 3-子群为循环, 从而及定理的假设推出 $Q$ 为循环, 推出 $G=Z(G) K$. 但由引理 4 知 $K<G$, 所以 $2 \mid\left(\left|G / G^{\prime}\right|,|Z(G)|\right)$. 再一次应用引理 2 得 $|\operatorname{Cent}(G)|_{2} \geqslant 2$, 而由引理 1 及 $\operatorname{Inn}(G)=G / Z(G)$ $\cong A_{4}$ 推出 $|\operatorname{Cent}(G)|_{2}=1$. 这矛盾证明了我们的断言, 而且可设 $Q$ 非循环, 故得 $Q \cong C_{3} \times C_{3}$. 于 是推出 $G$ 有 3 阶循环子群为其直因子, 与命题 2 的假设矛盾. (2) 证毕.

(3) $P$ 是交换群.

设 $P$ 非交换, 由 (2) 知 $2 \mid\left(\left|G / G^{\prime}\right|,|Z(G)|\right)$, 于是由引理 2 及结论 (1) 推出 $G / G^{\prime}$ 的 Sylow 2- 子 群循环, 从而推出 $P$ 也循环, 这是一个矛盾.

(4) $|G / Z(G)|_{q}=q^{2}$.

假设不真, 则 $|G / Z(G)|_{q}=q$, 故 $Q$ 为交换群, $Q=C_{Q}(P) \times[P, Q]$, 于是 $G=P[P, Q] \times C_{Q}(P)$. 由假设 $G$ 没有非平凡的交换直因子, 故交换群 $C_{Q}(P)=1$, 得出 $|Q|=q$. 特别地, 有 $|\operatorname{Cent}(G)|_{q}=1$. 令 $\hat{Q}$ 为 $\operatorname{Inn}(G)$ 的 Sylow $q$ - 子群, 那么 $\hat{Q} \cong Q \cong C_{q}$. 又令 $\hat{P}$ 为 $\operatorname{Inn}(G)$ 的 Sylow $p$ - 子群. 由 
Frattini 推理, $\operatorname{Aut}(G)=\operatorname{Inn}(G) N(\hat{P})=\hat{Q} N(\hat{P}), N(\hat{P})$ 表示 $\hat{P}$ 在 $\operatorname{Aut}(G)$ 中的正规化子, 且 $N(\hat{P})<$ $\operatorname{Aut}(G)$. 若 $\operatorname{Aut}(G)$ 为 $q-$ 闭, 那么 $N(\hat{P}) \cong \hat{P} \times C_{q}$, 推出 $\operatorname{Aut}(G)$ 的一个 $q$ 阶子群中心化 $\operatorname{lnn}(G)$, 由引理 $1|\operatorname{Cent}(G)|_{q} \neq 1$, 与上面的结果矛盾. 于是 $\operatorname{Aut}(G)$ 不能为 $q$-闭, 推出 $\operatorname{Aut}(G)$ 含子群 $\hat{A} \cong A_{4},|\operatorname{Aut}(G): \hat{A}|=3$. 由此推得 $\operatorname{Aut}(G) \cong A_{4} \times C_{q}$, 再一次与 $|\operatorname{Cent}(G)|_{q}=1$ 矛盾. 这证明了 (4) .

(5) $|G / Z(G)|=2 q^{2}$.

只需证明 $G$ 有 2 阶外自同构. 假设无, 由假设有 $|G / Z(G)|=4 q^{2}$. 由 (3), $P$ 是交换 2- 群, 推出 $G / Z(G)$ 的中心不含 2 阶元. 由引理 1 得出 $|\operatorname{Cent}(G)|_{2}=1$, 从而由引理 2 推出 $2 \nmid|Z(G)|$, 故 有 $C_{P}(Q)=1$. 如果 $|Q| \geqslant q^{3}$, 那么 $Z(G) \cap Q>1$. 同时, 由引理 $5 Q$ 可以表为一个交换群 $A$ 借助 一个循环群 $B$ 的半直积, 故 $Q$ 有 2 阶自同构 $\alpha: a b \rightarrow a^{-1} b \forall a \in A ; b \in B$, 且限制 $\alpha_{z} \neq 1$, 这里 $Z=Z(G) \cap Q$. 应用文献 [7, 引理 7], $G$ 有 2 阶外自同构, 矛盾. 于是有 $|Q|=q^{2}$. 若 $Q$ 为循 环, 则 $Q$ 的每个自同构可以扩张为 $G$ 的自同构, 推出 $G$ 有 $q$ 阶外自同构, 同样不可. 因此得 $Q \cong C_{q} \times C_{q}$, 由引理 3 得出矛盾: $2^{3} \| \operatorname{Aut}(G) \mid$. (5) 证完.

(6) $P \cong C_{2}$ 或 $C_{4}$.

设 $|P|>2$, 由 $(5), 2 \| Z(G) \mid$, 又因 $P$ 为交换群, 应用引理 2 及结论 $(1)$, 得 $P \cong C_{4}$.

(7) $Q$ 非交换 .

设 $Q$ 为交换, 则 $Q=C_{Q}(P) \times[P, Q]$, 于是 $G=P[P, Q] \times C_{Q}(P)$. 由假设交换直因子 $C_{Q}(P)=1$, 故得 $Q \cong C_{q} \times C_{q}$ (因为 $Q$ 不能为循环). 如果我们又能证明 $Z(G)=1$, 则由引理 3 获得一个矛 盾, (7) 的证明便完成. 下面来证 $Z(G)=1$, 为此只需证 $P \cong C_{2}$.

由 (5) 知 $G$ 是超可解的, 故 $G$ 可表为半直积 $[\langle x\rangle] K,|x|=q, K=P\langle y\rangle, Q=\langle x\rangle \times\langle y\rangle$. 定 义 $\alpha: x a \mapsto x^{-1} a \forall a \in K$, 那么 $\alpha$ 是 $G$ 的一个 2 阶自同构. 现在假设 $P \neq C_{2}$, 则由 (6) 得 $P=$ $\langle u\rangle,|u|=4, u^{2} \in Z(G)$. 用 $I_{u}$ 表由 $u$ 诱导的内自同构, 那么 $x^{I_{u}}=x^{-1}, y^{I_{u}}=y^{-1}$ 且有 $\alpha I_{u}=I_{u} \alpha$, 所以 $\left\langle I_{u}, \alpha\right\rangle=\left\langle I_{u}\right\rangle \times\langle\alpha\rangle$ 是 $\operatorname{Aut}(G)$ 的一个 Sylow 2- 子群. 其次, 由引理 2 推出 $G$ 必有 2 阶中心自 同构, 比如 $\sigma$, 可设 $\sigma \in\left\langle I_{u}\right\rangle \times\langle\alpha\rangle$, 又 $I_{u}$ 和 $\alpha$ 显然都不是中心自同构, 故 $\sigma=\alpha I_{u}$, 于是有

$$
y Z(G)=y^{\alpha^{l}} Z(G)=y^{l^{\prime}} Z(G)=y^{-1} Z(G),
$$

得出 $y^{2} \in Z(G)$, 这显然不可. 于是 $P \cong C_{2}$ 成立并且 (7) 被证明.

(8) $G$ 不存在.

有 $Z(Q)=Z(G) \cap Q, Q / Z(Q)$ 为 $q^{2}$ 阶初等交换群, 由 Mashke 定理得

$$
Q / Z(Q)=A / Z(Q) \times B / Z(Q),
$$

右边两个直因子都是 $q$ 阶群且 $P$ - 不变. 显然有 $[P, A] \neq 1 \neq[P, B]$, 所以 $[P, A]=\langle x\rangle,[P, B]=$ $\langle y\rangle$ 都是 $q$ 阶元. 记 $K=P\langle y\rangle$, 则 $G=[A] K$. 因为 $A$ 为交换 $q$-群, 故对于每个与 $q$ 互素的整数 $m, G$ 有自同构 $\alpha_{m}: g a \mapsto g a^{m} \forall g \in K$ 及 $a \in A$. 若 $A$ 含 $q^{2}$ 阶元, 可选取 $m$ 使得 $\alpha_{m}$ 的阶为 $q$, 自然 $\alpha_{m}$ 在 $Z(Q)$ 上的限制 $\neq 1$, 因而是 $G$ 的一个 $q$ 阶外自同构, 不可. 这证明了 $A$ 必为初等交换 $q$ - 群. 类似的讨论还可得出 $q=3$.

1: 面的讨论得出 $Q=\langle x, y\rangle \times Z, Z \leqslant Z(Q) \leqslant Z(G)$. 因 $\langle x, y\rangle$ 为 $P$ - 不变, 有 $G=P\langle x, y\rangle \times$ $Z$, 由假设交换直因子 $Z=1$, 即 $G=\langle x, y\rangle, \Phi(Q) \leqslant Z(Q)$. 利用 $G$ 无非平凡交换直因子及上一 段的讨论还可得出 $\Phi(Q)=Z(Q)$, 从而知 $Q$ 为极小非交换 3-群, 它的生成元 $x$ 与 $y$ 都是 3 阶 元, 得出结论: $|Q|=3^{3}, \exp (Q)=3$. 
根据上面讨论的结果, 能够给出 $G$ 的定义关系如下:

$$
\begin{aligned}
G= & \langle a, x, y, z| a^{2}=x^{3}=y^{3}=z^{3}=1, \\
& {[x, y]=z,[a, z]=[x, z]=[y, z]=1, } \\
& \left.a^{-1} x a=x^{-1}, a^{-1} y a=y^{-1} .\right\rangle,
\end{aligned}
$$

其中 $n=1$ 或 2 . 记 $I_{a}$ 为 $a$ 诱导的内自同构, $\alpha$ 为如下定义的 2 阶外自同构:

$$
\alpha=\left(\begin{array}{llll}
a & x & y & z \\
a & x^{-1} & y & z^{-1}
\end{array}\right),
$$

那么 $\left\langle I_{a}\right\rangle \times\langle\alpha\rangle$ 是 $\operatorname{Aut}(G)$ 的一个 Sylow 2- 子群, 并且还是 $\left\langle I_{a}\right\rangle$ 在 $\operatorname{Aut}(G)$ 中的正规化子. 现在 定义 $\gamma$ 如下:

$$
\gamma=\left(\begin{array}{llcl}
a & x & y & z \\
a & x^{-1} & x^{-1} y^{-1} z & z
\end{array}\right)
$$

则 $\gamma$ 提供 $G$ 的一个自同构, 且 $\gamma^{-1} I_{a} \gamma=I_{a} I_{a^{\prime}}=I_{a}$, 即 $\gamma \in\left\langle I_{a}\right\rangle \times\langle\alpha\rangle$. 但显然有 $\alpha \neq \gamma \neq I_{a}$, 故 $\gamma=I_{a} \alpha$, 由此推出 $x^{-1}=x^{y}=x^{I_{\alpha} \alpha}=x$, 与 $x$ 为奇阶元矛盾. 这就证明了 (8).

定理 1 证完.

\section{参 考 文 献}

1 Iyer H K. On solving the equation $\operatorname{Aut}(X)=G$. Rocky Mauntain J Math, 1979, 9(4): 653 670

2 Machale D. Some finite groups which are rarely automorphism group. Proc R Ir Acad, 1983, 83A(2): $189 \sim 196$

3 Curran M J. Automorphisms of crtain p-groups (p odd). Bull Austral Hath Soc, 1988, 38(2): 299 305

4 Flym J, Machale D. Determing all finite groups whose automorphism group is a p-group. Proc R Ir Acad, 1991, 91A(2): $259 \sim 264$

5 黄安平. 自同构群阶为 $p^{2} q$ 的有限群. 科学通报, 1989, 34(3): 235

6 陈贵云. 自同构群阶为 $p_{1} p_{2} \cdots p_{n}$ 或 $p q^{2}$ 的有限群. 西南师范大学学报, 1990, 15(1): $21 \sim 27$

7 李世荣。某些有限群的自同构群，中国科学, A 辑, 1993, 23(12): 1276 1282

8 Sanders P R. The central automorphisms of a finite group. J London Math Soc, 1969, 44(2): $225 \sim 228$ 